\title{
Impregnated Copper(II) Oxide on Magnetite as Catalyst for the Synthesis of Benzo[b]furans from 2-Hydroxyarylcarbonyl derivatives and Alkynes
}

\author{
Juana M. Pérez, ${ }^{[a]}$ and Diego J. Ramón ${ }^{\star[a]}$
}

\begin{abstract}
The coupling-allenylation-cyclization of 0 hydroxybenzaldehydes with alkynes in the presence of 4methylbenzenesulfonohydrazide is carried out using the heterogeneous impregnated copper(II) oxide on magnetite catalyst and ethanol, as non-toxic and bio-renewable solvent. The catalyst can be removed easily from the reaction medium by a simply magnetic decantation, performing the reaction with a very low copper loading. The in situ reduction of starting nanoparticles of $\mathrm{Cu}(\mathrm{II})$ oxide to sheets of $\mathrm{Cu}(0)$ made impossible the recyclability of catalyst. The versatility of the reaction was proved by expansion to different o-hydroxyacetophenones giving 2,3-disubstituted benzo[b]furans. The reaction could be scale up to gram scale without any noticeable problem.
\end{abstract}

$N$-tosylhydrazones ${ }^{[7]}$ catalyzed by copper salts (CuBr, $\left.10 \mathrm{~mol} \%\right)$ in acetonitrile gave directly the corresponding benzo[b]furans. An alternative route was designed, avoiding the high cost of the ortho-functionalized arylalkynes, starting from the corresponding $\mathrm{N}$-tosylhydrazone, using terminal alkynes, acetonitrile as solvent, and the homogeneous $\mathrm{CuBr}(10 \mathrm{~mol} \%)$ as catalyst. ${ }^{[8]}$

Herein, we report the first example of heterogeneous catalysis by $\mathrm{CuO}-\mathrm{Fe}_{3} \mathrm{O}_{4}{ }^{\left[{ }^{9]}\right.}$ for the coupling-allenylation-cyclization of $o$ hydroxybenzaldehydes with terminal alkynes yielding benzo[b]furans derivatives.

\section{Results and Discussion}

The coupling of 2-hydroxybenzaldehyde (1a) with phenylacetylene (2a) using impregnated copper(II) oxide on magnetite, in the presence of 4-methylbenzenesulfonohydrazide, was selected as the model for the optimization of the reaction conditions (Table 1). Initially, the reaction was studied with different solvents (entries 1-8) and in its absence (entry 9), obtaining only good yield when $\mathrm{EtOH}$ was used. After that, some organic and inorganic bases were examined (entries 10-18) obtaining traces of the product with organic bases and moderate yields when some inorganic hydroxide salts were used. The reaction failed in the absence of base (entry 19). Furthermore, the decrease of the temperature on the reaction was tested (entries 20 and 21) obtaining lower yields. The reaction with only one equivalent of base (entry 22) was tested obtaining a decrease in the reaction yield. Moderate results were obtained using three equivalents of terminal alkyne (entry 23 ) or using different amounts of solvent (entries 24 and 25).

Having stablished the optimal reaction conditions, different catalysts prepared by simple impregnation protocol were tested on the reaction (Table 2). ${ }^{[10]}$ First of all, the reaction was tested without catalyst (entry 1) and only with the support of the catalyst (entries 2 and 3 ) the reaction did not take place. After that, different supported metal catalysts were probed (entries 416), obtaining traces of the product with some of them. In the case of the palladium catalyst, the reaction took place with moderate results. Having stablished that the impregnated copper(II) oxide on magnetite was the best catalyst to perform this reaction (entry 6), different amounts of them were probed (entries 17 and 18). When trying to decrease the metal loading the reaction took place with moderate results. Then, a variety of copper(I) and copper(II) salts were used obtaining moderates yields (entries 19-25), and confirming the higher activity of the supported catalyst. 
Table 1. Optimization of the Reaction Conditions ${ }^{[\mathrm{a}]}$

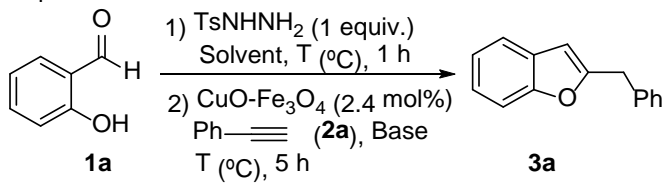

\begin{tabular}{|c|c|c|c|c|}
\hline Entry & Solvent & Base & $\mathrm{T}\left({ }^{\circ} \mathrm{C}\right)$ & Yield $^{[\mathrm{b}]}$ \\
\hline 1 & THF & $\mathrm{Cs}_{2} \mathrm{CO}_{3}$ & 100 & 26 \\
\hline 2 & Toluene & $\mathrm{Cs}_{2} \mathrm{CO}_{3}$ & 100 & 0 \\
\hline 3 & $\mathrm{CH}_{3} \mathrm{CN}$ & $\mathrm{Cs}_{2} \mathrm{CO}_{3}$ & 100 & 0 \\
\hline 4 & 1,4-Dioxane & $\mathrm{Cs}_{2} \mathrm{CO}_{3}$ & 100 & 3 \\
\hline 5 & DMF & $\mathrm{Cs}_{2} \mathrm{CO}_{3}$ & 100 & 0 \\
\hline 6 & $\mathrm{H}_{2} \mathrm{O}$ & $\mathrm{Cs}_{2} \mathrm{CO}_{3}$ & 100 & 0 \\
\hline 7 & DMSO & $\mathrm{Cs}_{2} \mathrm{CO}_{3}$ & 100 & 0 \\
\hline 8 & $\mathrm{EtOH}$ & $\mathrm{Cs}_{2} \mathrm{CO}_{3}$ & 100 & 91 \\
\hline 9 & - & $\mathrm{Cs}_{2} \mathrm{CO}_{3}$ & 100 & 0 \\
\hline 10 & $\mathrm{EtOH}$ & KOAC & 100 & 0 \\
\hline 11 & $\mathrm{EtOH}$ & $\mathrm{MeONa}$ & 100 & 3 \\
\hline 12 & $\mathrm{EtOH}$ & $\mathrm{Et}_{3} \mathrm{~N}$ & 100 & 0 \\
\hline 13 & $\mathrm{EtOH}$ & DABCO & 100 & 0 \\
\hline 14 & $\mathrm{EtOH}$ & $\mathrm{KOH}$ & 100 & 56 \\
\hline 15 & $\mathrm{EtOH}$ & $\mathrm{NaOH}$ & 100 & 43 \\
\hline 16 & $\mathrm{EtOH}$ & $\mathrm{CsOH} \cdot \mathrm{H}_{2} \mathrm{O}$ & 100 & 58 \\
\hline 17 & $\mathrm{EtOH}$ & tBuOK & 100 & 34 \\
\hline 18 & $\mathrm{EtOH}$ & $\mathrm{K}_{2} \mathrm{CO}_{3}$ & 100 & 0 \\
\hline 19 & EtOH & - & 100 & 0 \\
\hline 20 & $\mathrm{EtOH}$ & $\mathrm{Cs}_{2} \mathrm{CO}_{3}$ & 50 & 28 \\
\hline 21 & $\mathrm{EtOH}$ & $\mathrm{Cs}_{2} \mathrm{CO}_{3}$ & $\mathrm{RT}$ & 37 \\
\hline $22^{[\mathrm{c}]}$ & $\mathrm{EtOH}$ & $\mathrm{Cs}_{2} \mathrm{CO}_{3}$ & 100 & 35 \\
\hline $23^{[\mathrm{d}]}$ & $\mathrm{EtOH}$ & $\mathrm{Cs}_{2} \mathrm{CO}_{3}$ & 100 & 63 \\
\hline $24^{[\mathrm{e}]}$ & $\mathrm{EtOH}$ & $\mathrm{Cs}_{2} \mathrm{CO}_{3}$ & 100 & 58 \\
\hline $25^{[f]}$ & $\mathrm{EtOH}$ & $\mathrm{Cs}_{2} \mathrm{CO}_{3}$ & 100 & 44 \\
\hline
\end{tabular}

[a] Reaction carried out using $0.4 \mathrm{mmol} \mathbf{1 a}, 0.5 \mathrm{mmol} 2 \mathrm{a}, 1.2 \mathrm{mmol}$ of base in $2 \mathrm{~mL}$ of the corresponding solvent. [b] Yield calculated by GC using tridecane as an internal standard. [c] Reaction carried out using $0.4 \mathrm{mmol}$ 1a, $0.5 \mathrm{mmol} 2 \mathrm{a}, 0.4 \mathrm{mmol}$ of base in $2 \mathrm{~mL}$ of the corresponding solvent. [d] Reaction carried out using $0.4 \mathrm{mmol} \mathbf{1 a}, 1.2 \mathrm{mmol} \mathbf{2 a}, 1.2 \mathrm{mmol}$ of base in 2 $\mathrm{mL}$ of the corresponding solvent. [e] Reaction carried out using $0.4 \mathrm{mmol} \mathbf{1 a}$, $0.5 \mathrm{mmol} 2 \mathrm{a}, 1.2 \mathrm{mmol}$ of base in $1 \mathrm{~mL}$ of the corresponding solvent. [f] Reaction carried out using $0.4 \mathrm{mmol} 1 \mathbf{a}, 0.5 \mathrm{mmol} 2 \mathbf{a}, 1.2 \mathrm{mmol}$ of base in 6 $\mathrm{mL}$ of the corresponding solvent.
Table 2. Optimization of the Catalyst ${ }^{[\mathrm{a}]}$<smiles>O=Cc1ccccc1O</smiles>

1) $\mathrm{TsNHNH}_{2}$ (1 equiv.)

$\mathrm{EtOH}(2 \mathrm{~mL}), 100^{\circ} \mathrm{C}, 1 \mathrm{~h}$

2) Catalyst (mol\%)

$\mathrm{Ph}=(2 \mathrm{a})$

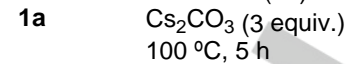

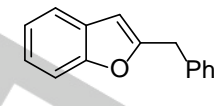

$3 a$

\begin{tabular}{|c|c|c|}
\hline Entry & Catalyst (mol \%) & Yield $(\%)^{[b]}$ \\
\hline 1 & & 5 \\
\hline 2 & nano- $\mathrm{Fe}_{3} \mathrm{O}_{4}(162)$ & 4 \\
\hline 3 & micro- $\mathrm{Fe}_{3} \mathrm{O}_{4}(162)$ & 4 \\
\hline 4 & $\mathrm{CoO}-\mathrm{Fe}_{3} \mathrm{O}_{4}(3.54)$ & 4 \\
\hline 5 & $\mathrm{NiO}-\mathrm{Fe}_{3} \mathrm{O}_{4}(2.58)$ & 3 \\
\hline 6 & $\mathrm{CuO}-\mathrm{Fe}_{3} \mathrm{O}_{4}(2.4)$ & 91 \\
\hline 7 & $\mathrm{Ru}_{2} \mathrm{O}_{3}-\mathrm{Fe}_{3} \mathrm{O}_{4}$ (3.3) & 4 \\
\hline 8 & $\mathrm{Rh}_{2} \mathrm{O}_{3}-\mathrm{Fe}_{3} \mathrm{O}_{4}(1.05)$ & 4 \\
\hline 9 & $\mathrm{PdO}-\mathrm{Fe}_{3} \mathrm{O}_{4}(3.04)$ & 32 \\
\hline 10 & $\mathrm{AgO}_{2} / \mathrm{Ag}-\mathrm{Fe}_{3} \mathrm{O}_{4}(3.13)$ & 0 \\
\hline 11 & $\mathrm{OsO}_{2}-\mathrm{Fe}_{3} \mathrm{O}_{4}(1.28)$ & 3 \\
\hline 12 & $\mathrm{PtO} / \mathrm{PtO}_{2}-\mathrm{Fe}_{3} \mathrm{O}_{4}(1.34)$ & 4 \\
\hline 13 & $\mathrm{Au}_{2} \mathrm{O}_{3} / \mathrm{Au}-\mathrm{Fe}_{3} \mathrm{O}_{4}(0.35)$ & 4 \\
\hline 14 & $\mathrm{PdO} / \mathrm{Cu}-\mathrm{Fe}_{3} \mathrm{O}_{4}(3.82 / 2.24)$ & 6 \\
\hline 15 & $\mathrm{NiO} / \mathrm{Cu}-\mathrm{Fe}_{3} \mathrm{O}_{4}(2.28 / 2.20)$ & 2 \\
\hline 16 & $\mathrm{WO}_{3}-\mathrm{Fe}_{3} \mathrm{O}_{4}(1.41)$ & 2 \\
\hline 17 & $\mathrm{CuO}-\mathrm{Fe}_{3} \mathrm{O}_{4}(1.2)$ & 56 \\
\hline 18 & $\mathrm{CuO}-\mathrm{Fe}_{3} \mathrm{O}_{4}(0.5)$ & 44 \\
\hline 19 & $\mathrm{CuO}(2.4)$ & 46 \\
\hline 20 & $\mathrm{Cu}(\mathrm{OAc})_{2}(2.4)$ & 43 \\
\hline 21 & $\mathrm{CuBr}_{2}(2.4)$ & 46 \\
\hline 22 & $\mathrm{Cu}_{2} \mathrm{O}(2.4)$ & 36 \\
\hline 23 & CuCN (2.4) & 49 \\
\hline 24 & $\mathrm{CuBr}(2.4)$ & 61 \\
\hline 25 & $\mathrm{Cu}$ (powder) (2.4) & 9 \\
\hline
\end{tabular}

[a] Reaction carried out using $0.4 \mathrm{mmol} \mathbf{1 a}$ and $0.5 \mathrm{mmol}$ 2a. [b] Yield calculated by GC using tridecane as an internal standard.

Once the optimal conditions were obtained, the problem of recycling was faced. When the catalyst was recovered from the reaction mixture by using a magnet, washed with ethanol and reused under the same reaction conditions only traces $(12 \%)$ of 
the expected product $\mathbf{3 a}$ were obtained (see Figure 3). To explain these phenomena some studies were carried out. First of all, X-ray photoelectron spectroscopy before and after the reaction (Figure 1 ). Showing that before the reaction the catalyst was formed by a mixture $4: 1$ of $\mathrm{Cu}(\mathrm{II}) \mathrm{Cu}(0)$ nanoparticles, and after the reaction only $\mathrm{Cu}(0)$ was detected in the catalyst.
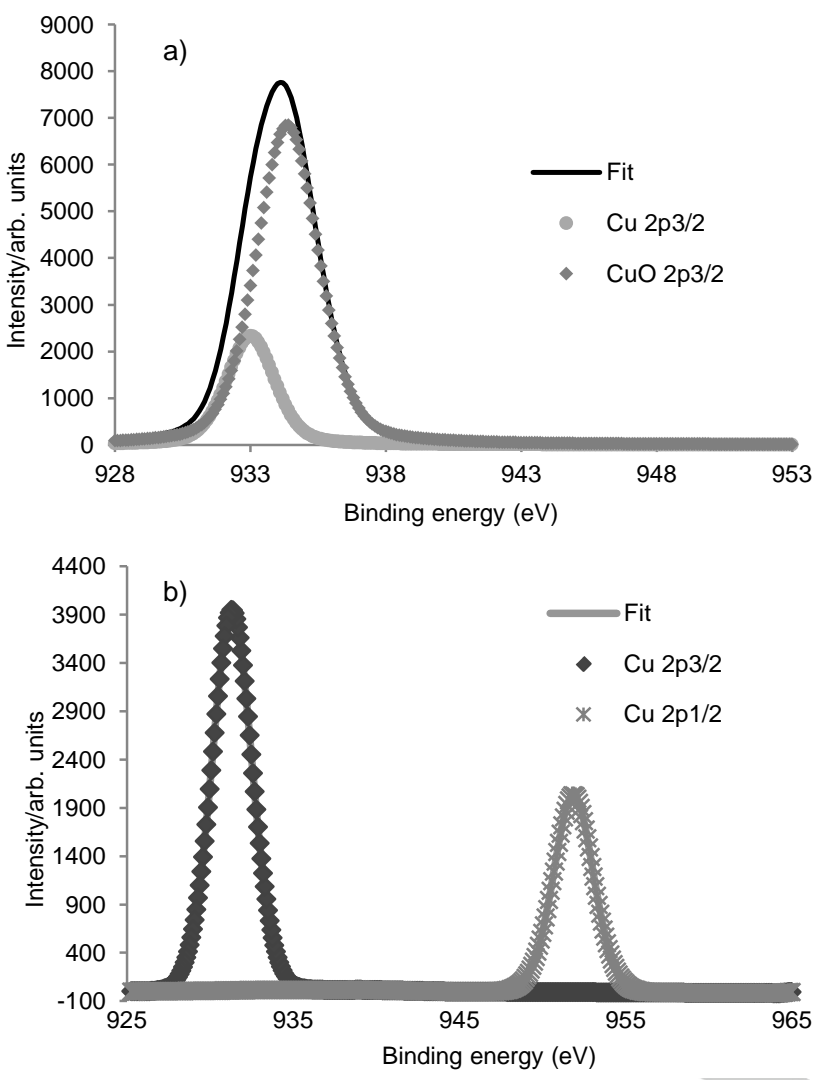

Figure 1. a) XPS of copper catalyst before reaction. b) XPS of copper catalyst after reaction.

Transmission Electron Microscopy (TEM) images were analyzed before and after complete the reaction, obtaining that before reaction $73 \%$ of the copper oxide nanoparticles had an average size between 2-6 nm and after completion of the reaction we can not observed the nano-structure particles, and they were transformed into a homogeneous sheet of copper(0) covering the magnetite surface (Figure 2).

The inductively coupled plasma-mass spectroscopy (ICP-MS) analysis of the reaction solution showed the presence of copper (20.5\% of the initial amount) as well as a low amount of iron (0.03\% of the initial amount). With these results in hand we could speculate that the heterogeneous $\mathrm{CuO}-\mathrm{Fe}_{3} \mathrm{O}_{4}$ catalyst could serve as a reservoir of copper and when the copper goes to the reaction solution ${ }^{[11]}$ catalyzes the reaction and after that it is reduced by ethanol to give a $\mathrm{Cu}(0)$ sheet. In fact, the obtained result using $\mathrm{Cu}(0)$ powder (entry 25 , Table 2 ) was the same as the one using the recycled catalyst.
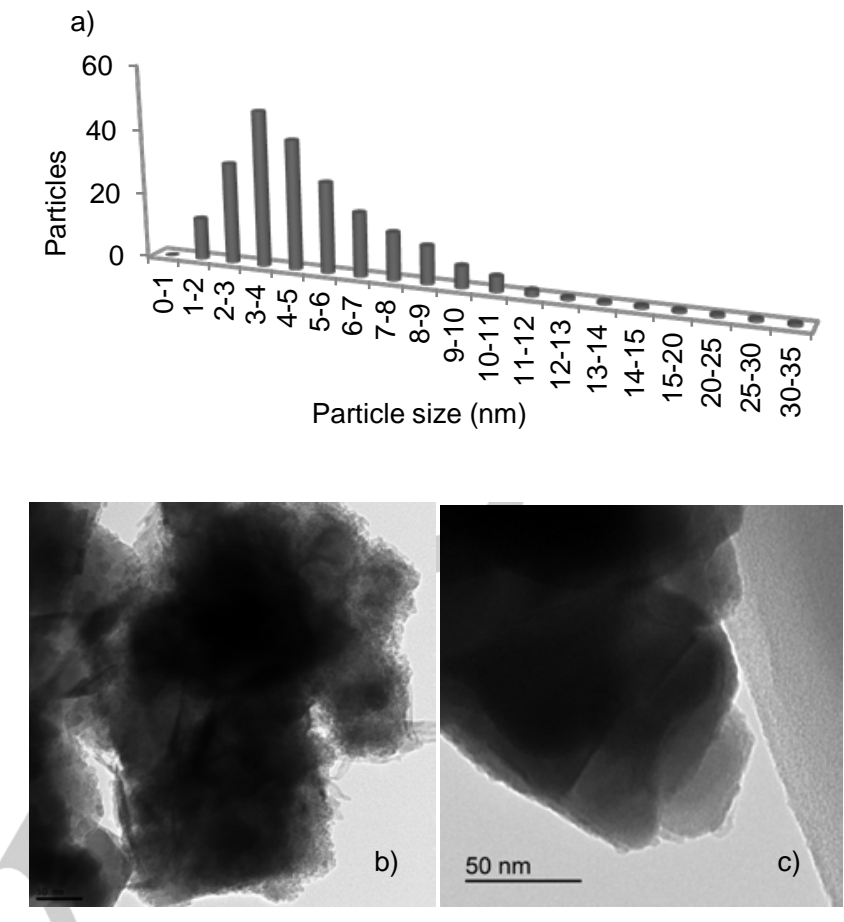

Figure 2. a) Copper particle size distribution on catalyst before reaction. b) TEM image of copper catalyst before reaction. c) TEM image of copper catalyst after reaction.

The hot filtration experiment was performed to confirm that nanoparticles of copper were leached to the homogeneous solution that, after completion of the standard reaction, the catalyst was removed and the same reaction was tried to be performed but in this case 1-ethynyl-4-methoxybenzene was used. After $6 \mathrm{~h}$ the reaction was quenched and only traces of the corresponding product $\mathbf{3} \mathbf{j}$ were observed by ${ }^{1} \mathrm{H}-\mathrm{NMR}$, recovering the starting alkyne unchanged.

Due to the inability of the catalyst to be reused after the completion of the reaction, re-oxidation was tried using different methodologies. One of the methodologies used to oxidize the $\mathrm{Cu}(0)$ sheet formed was bubbling up the catalyst with $\mathrm{O}_{2}$ during 4 hours using THF as solvent, but only $22 \%$ of yield could be obtained when the reaction was repeated. Another method used was to add $t \mathrm{BuOOH}$ in decane to the catalyst, previously washed with ethanol to remove the crude of the reaction, and the mixture was heated at $70{ }^{\circ} \mathrm{C}$ overnight, obtaining better results than the aforementioned protocol, but it was not enough to recycle the catalyst with good results (Figure 3).

The hypothesized mechanism for the formation of the final product are similar to the previously introduced, ${ }^{[8]}$ as shown in Scheme 1. First of all, 2-hydroxybenzaldehyde reacts with 4methylbenzenesulfonohydrazine to give the corresponding hydrazone, which in turn reacts with the base $\left(\mathrm{Cs}_{2} \mathrm{CO}_{3}\right)$ to form 2-(diazomethyl)phenol derivative. 


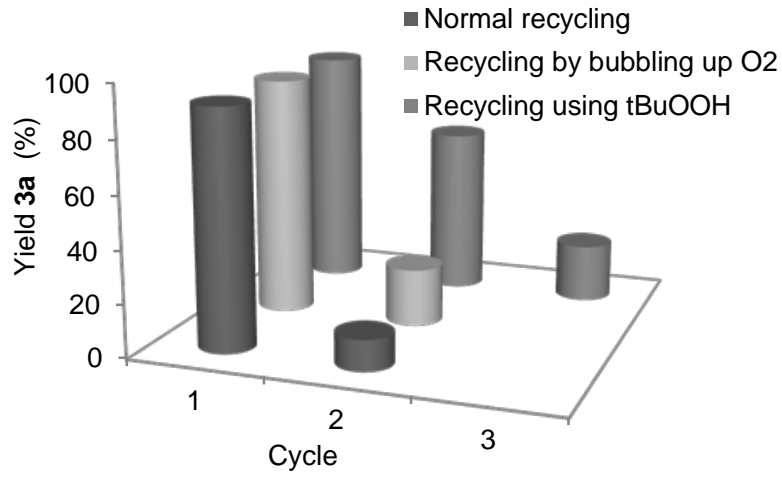

Figure 3. Catalyst recycling using different methodologies.

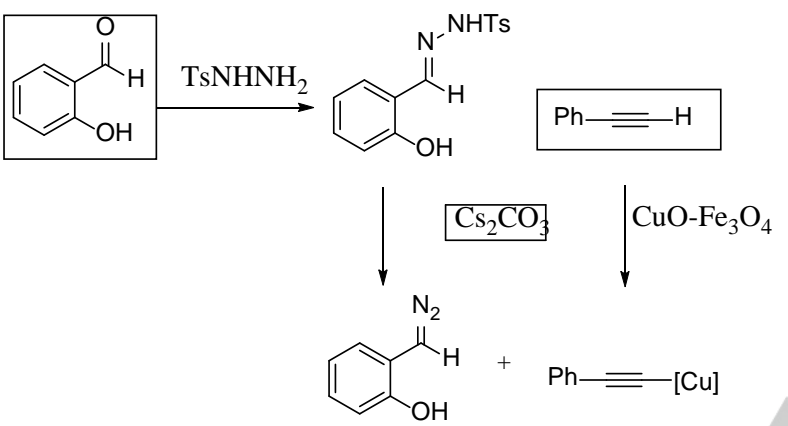<smiles>CC(C)CCCCCCCC(C)(C)c1ccccc1O</smiles><smiles>CCC(C)C(C)(CC)CC(C)O</smiles><smiles>Oc1ccccc1C=C=Cc1ccccc1</smiles>

[Cu]<smiles>CC(=Cc1ccccc1O)C(Cl)(Cl)c1ccccc1</smiles><smiles>CCCC(C)CC(C)C(=O)O</smiles><smiles>ClC(Cl)(c1ccccc1)C(Cl)(Cl)c1cc2ccccc2o1</smiles><smiles>CCOCC1CCCC1(CC)C(=O)O</smiles>
$[\mathrm{Cu}]$

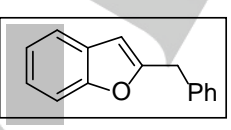

Scheme 1. Proposed mechanism for the synthesis of benzo[b]furans.

At the same time, phenylacetylene reacts with the base giving copper phenyl acetylide. Although the hot filtration test seems to indicate that the reaction is heterogeneous, the big amount of leached copper could indicate the possible homogeneous pathway. Having formed these two species, they react with each other through a dediazotization reaction giving a copper carbene intermediate that through a migratory insertion forms [1-(2hydroxyphenyl)-3-phenylprop-2-yn-1-yl]copper intermediate. The subsequent protonation of this intermediate gives the corresponding allene, and finally it cycles generating the corresponding benzo[b]furan.

To check if the reaction took place through the formation of the $N$-tosylhydrazone, the reaction was performed starting from the previously prepared 4-methylbenzenesulfonohydrazine obtaining the product $\mathbf{3} \mathbf{a}$ with the same yield than under standard reaction conditions. Moreover, when only $30 \mathrm{~mol} \%$ of $\mathrm{TsNHNH}_{2}$ was used in the reaction, only $29 \%$ of conversion to product $3 \mathbf{a}$ was obtained. These results seem to indicate that the formation of hydrazine is fundamental in the reaction pathway.

The optimized reaction conditions were applied to other substrates (Table 3).

Table 3. Scope of the reaction ${ }^{[a]}$

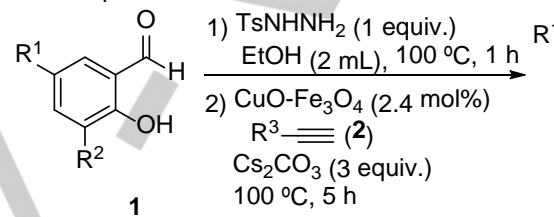

1 $100^{\circ} \mathrm{C}, 5 \mathrm{~h}$<smiles>[R]Cc1cc2cc([Y1])cc([R])c2o1</smiles>

\begin{tabular}{|c|c|c|c|c|c|}
\hline Entry & $\mathrm{R}^{1}$ & $\mathrm{R}^{2}$ & $\mathrm{R}^{3}$ & $\mathrm{~N}^{\circ}$ & $\begin{array}{l}\text { Yield } \\
(\%)^{[b]}\end{array}$ \\
\hline 1 & $\mathrm{H}$ & $\mathrm{H}$ & $\mathrm{Ph}$ & $3 a$ & $91(58)^{[\mathrm{c}]}$ \\
\hline 2 & $t \mathrm{Bu}$ & $t \mathrm{Bu}$ & $\mathrm{Ph}$ & $3 b$ & 71 \\
\hline 3 & $\mathrm{Br}$ & $\mathrm{OMe}$ & $\mathrm{Ph}$ & $3 c$ & 75 \\
\hline 4 & $\mathrm{H}$ & $\mathrm{H}$ & $2-\mathrm{BrC}_{6} \mathrm{H}_{4}$ & $3 d$ & 70 \\
\hline 5 & $\mathrm{H}$ & $\mathrm{H}$ & $3-\mathrm{ClC}_{6} \mathrm{H}_{4}$ & $3 e$ & 82 \\
\hline 6 & $\mathrm{H}$ & $\mathrm{H}$ & $4-\mathrm{BrC}_{6} \mathrm{H}_{4}$ & $3 f$ & 90 \\
\hline 7 & $\mathrm{H}$ & $\mathrm{H}$ & $4-\mathrm{CF}_{3} \mathrm{C}_{6} \mathrm{H}_{4}$ & $3 g$ & 92 \\
\hline 8 & $\mathrm{H}$ & $\mathrm{H}$ & $2-\mathrm{MeC}_{6} \mathrm{H}_{4}$ & $3 \mathrm{~h}$ & 67 \\
\hline 9 & $\mathrm{H}$ & $\mathrm{H}$ & $3-\mathrm{MeC}_{6} \mathrm{H}_{4}$ & $3 \mathbf{i}$ & 67 \\
\hline 10 & $\mathrm{H}$ & $\mathrm{H}$ & $4-\mathrm{MeOC}_{6} \mathrm{H}_{4}$ & $3 \mathbf{j}$ & 68 \\
\hline 11 & $\mathrm{H}$ & $\mathrm{H}$ & $\mathrm{CH}_{3}\left(\mathrm{CH}_{2}\right)_{4}$ & $3 k$ & 54 \\
\hline 12 & $\mathrm{H}$ & $\mathrm{H}$ & $\mathrm{C}_{6} \mathrm{H}_{11}$ & 31 & 39 \\
\hline 13 & $\mathrm{H}$ & $\mathrm{H}$ & $\mathrm{Cl}\left(\mathrm{CH}_{2}\right)_{3}$ & $3 m$ & 70 \\
\hline 14 & $\mathrm{H}$ & $\mathrm{H}$ & $\mathrm{CH}_{2} \mathrm{OC}_{5} \mathrm{H}_{10} \mathrm{O}$ & $3 n$ & 90 \\
\hline 15 & $\mathrm{H}$ & $\mathrm{H}$ & $3-(\mathrm{CH} \equiv \mathrm{C}) \mathrm{C}_{6} \mathrm{H}_{4}$ & 30 & 17 \\
\hline 16 & $t \mathrm{Bu}$ & $t \mathrm{Bu}$ & $4-\mathrm{BrC}_{6} \mathrm{H}_{4}$ & $3 p$ & 69 \\
\hline
\end{tabular}

[a] Reaction carried out using $0.4 \mathrm{mmol} \mathbf{1 a}$ and $0.5 \mathrm{mmol} 2 \mathrm{2a}$. [b] Isolated yield after column chromatography. [c] Reaction carried out using $8 \mathrm{mmol} \mathbf{1 a}$ and 10 $\mathrm{mmol} 2 \mathrm{a}$. 
Different substituted o-hydroxybenzaldehydes were used (entries 2 and 3) obtaining good results. After that some arylalkynes with electron-withdrawing substituents in different positions at the aromatic ring were tested (entries 4-7), obtaining good results. Slightly lower yields were obtained when the reaction was performed using arylalkynes with electron-donating groups at the aromatic ring (entries 8-10). Then, some aliphatic alkynes were tested (entries 11-14) obtaining moderates to good yields. The reaction could be performed selectively using a diyne giving the mono benzofuran $\mathbf{2 0}$ in low yield (entry 15). To finish with the reaction scope a combination of substituted 0 hydroxybenzaldehyde and a substituted alkyne were used to obtain compound $3 p$ with good yield (entry 16). The reaction could be scaled up to 20 fold obtaining good yield (entry 1 , footnote c).

The same reaction could be carried out with different ohydroxybenzophenones, reaching good yields of the corresponding substituted benzo[b]furan in both cases, showing the great versatility of the reaction (Scheme 2 ).<smiles>[R]c1ccc(O)c(C(C)=O)c1</smiles>

$4 a \mathrm{R}=\mathrm{H}$ 4b $\mathrm{R}=\mathrm{Br}$
1) $\mathrm{TSNHNH}_{2}$ (1 equiv.) $\mathrm{EtOH}(2 \mathrm{~mL}), 100^{\circ} \mathrm{C}, 1 \mathrm{~h}$ 2) $\mathrm{CuO}-\mathrm{Fe}_{3} \mathrm{O}_{4}(2.4 \mathrm{~mol} \%)$ $\mathrm{Ph}=(2 \mathrm{a})$ $\mathrm{Cs}_{2} \mathrm{CO}_{3}$ (3 equiv.) $100^{\circ} \mathrm{C}, 5 \mathrm{~h}$

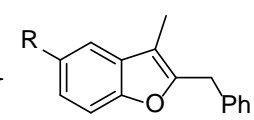

$5 \mathbf{a} \mathrm{R}=\mathrm{H}(62 \%)$ $5 b \mathrm{R}=\mathrm{Br}(57 \%)$
Scheme 2. Reaction with o-hydroxyacetophenones.

\section{Conclusions}

In conclusion, we have demonstrated that impregnated copper(II) oxide on magnetite is a cheap and versatile catalyst for the coupling-allenylation-cyclization reaction of 0 hydroxybenzaldehydes, (or ketones), and alkynes using ethanol as non-toxic and bio-renewable solvent and in the presence of 4-methylbenzenesulfonohydrazide. The magnetic properties of the catalyst allow its separation from the reaction medium very easily. The high activity of heterogeneous copper catalyst permitted the use of a very small amount. This new approach is quite general permiting the construction of benzofuran substituted at 2-, and 3-positions.

\section{Experimental Section}

General Information

XPS analyses were carried out on a VG-Microtech Mutilab. TEM images were obtained on a JEOL, model JEM-2010 equipped with an X-ray detector OXFORD INCA Energy TEM 100 for microanalysis (EDS). XRF analyses were obtained on a PHILIPS MAGIX PRO (PW2400) X-ray spectrometer equipped with a rhodium $X$-ray tube and a beryllium window. BET isotherms were carried out on a AUTOSORB-6 (Quantachrome), using $\mathrm{N}_{2}$. Melting points were obtained with a Reichert Thermovar apparatus. NMR spectra were recorded on a Bruker AC-300 (300 MHz for ${ }^{1} \mathrm{H}$ and $75 \mathrm{MHz}$ for ${ }^{13} \mathrm{C}$ ) using $\mathrm{CDCl}_{3}$ as a solvent and TMS as internal standard for ${ }^{1} \mathrm{H}$ and ${ }^{13} \mathrm{C}$; chemical shifts are given in $\delta$ (parts per million) and coupling constants $(J)$ in Hertz. FT-IR spectra were obtained on a JASCO 4100LE (Pike Miracle ATR) spectrophotometer. Mass spectra (EI) were obtained at $70 \mathrm{eV}$ on a Himazdu QP-5000 spectrometer, giving fragment ions in $\mathrm{m} / \mathrm{z}$ with relative intensities (\%) in parentheses. The chromatographic analyses (GLC) were determined with a Hewlett Packard HP-5890 instrument equipped with a flame ionization detector and $12 \mathrm{~m} \mathrm{HP}-1$ capillary column $(0.2 \mathrm{~mm}$ diam, 0.33 $\mathrm{mm}$ film thickness, OV-1 stationary phase), using nitrogen $(2 \mathrm{~mL} / \mathrm{min})$ as a carrier gas, $T_{\text {injector }}=275^{\circ} \mathrm{C}, T_{\text {detector }}=300^{\circ} \mathrm{C}, T_{\text {column }}=60^{\circ} \mathrm{C}(3 \mathrm{~min})$ and $60-270^{\circ} \mathrm{C}\left(15^{\circ} \mathrm{C} / \mathrm{min}\right), P=40 \mathrm{kPa}$. Thin layer chromatography (TLC) was carried out on Schleicher \& Schuell F1400/LS 254 plates coated with a $0.2 \mathrm{~mm}$ layer of silica gel; detection by $\mathrm{UV}_{254}$ light, staining with phosphomolybdic acid [25 g phosphomolybdic acid, $10 \mathrm{~g} \mathrm{Ce}\left(\mathrm{SO}_{4}\right)_{2} 4$ $\mathrm{H}_{2} \mathrm{O}, 60 \mathrm{~mL}$ of concentrated $\mathrm{H}_{2} \mathrm{SO}_{4}$ and $940 \mathrm{~mL} \mathrm{H}_{2} \mathrm{O}$ ]. Column chromatography was performed using silica gel 60 of $40-63$ mesh. All reagents were commercially available (Acros, Aldrich, Fluorochem) and were used as received. The ICP-MS analyses were carried out on a Thermo Elemental VGPQ-ExCell spectrometer. The Elemental Analysis was performed on an Elemental Microanalyzer Thermo Finningan Flash 1112 Series.

\section{General Procedure for the Preparation of Catalysts}

Commercially available $\mathrm{Fe}_{3} \mathrm{O}_{4}\left(<5 \mu \mathrm{m}\right.$, BET area: $9.86 \mathrm{~m}^{2} / \mathrm{g} ; 4 \mathrm{~g}, 17$ $\mathrm{mmol})$ was added to a stirred solution of the appropriate metal chloride $\mathrm{MCl}_{\mathrm{n}}(1 \mathrm{mmol})$ [or a mixture of $\mathrm{M}_{1} \mathrm{Cl}_{\mathrm{n}}(1 \mathrm{mmol})$ and $\mathrm{M}_{2} \mathrm{Cl}_{\mathrm{n}}$ (1 mmol) for bimetallic species] in deionized $\mathrm{H}_{2} \mathrm{O}(120 \mathrm{~mL})$. In the cases of the $\mathrm{Pd}$ and $\mathrm{Cu}-\mathrm{Pd}$ catalysts, a large excess of $\mathrm{KCl}(1 \mathrm{~g}, 13 \mathrm{mmol})$ was also added to the initial solution to increase the solubility of the $\mathrm{PdCl}_{2}$. After $10 \mathrm{~min}$ at r.t., the mixture was slowly basified with $1 \mathrm{M}$ aq $\mathrm{NaOH}$ to a $\mathrm{pH}$ of about 13. The mixture was then stirred for 1 day at r.t. under air. The catalyst was collected by filtration, washed with deionized $\mathrm{H}_{2} \mathrm{O}(3 \times 10 \mathrm{~mL})$, and dried for $24 \mathrm{~h}$ at $100{ }^{\circ} \mathrm{C}$ in a standard glassware oven.

\section{General procedure for the preparation of the products 3 .}

To a stirred solution of the corresponding aldehyde $(1,0.4 \mathrm{mmol})$ in $\mathrm{EtOH}$ $(2 \mathrm{~mL})$ was added 4-methylbenzenesulfonohydrazide $(74 \mathrm{mg}, 0.4 \mathrm{mmol})$ and the reaction was stirred at $100{ }^{\circ} \mathrm{C}$ during $1 \mathrm{~h}$. After that time, $\mathrm{Cs}_{2} \mathrm{CO}_{3}(390 \mathrm{mg}, 1.2 \mathrm{mmol})$, tridecane $(73.7 \mathrm{mg}, 0.4 \mathrm{mmol}$ as an internal standard), $\mathrm{CuO}-\mathrm{Fe}_{3} \mathrm{O}_{4}(50 \mathrm{mg}, 2.4 \mathrm{~mol} \%)$ were added to the reaction solution followed by the corresponding terminal alkyne $(2,0.5$ $\mathrm{mmol})$. The resulting mixture was stirred at $100^{\circ} \mathrm{C}$ during 5 hours. The catalyst was removed by a magnetic decantation and the solvent was removed under reduce pressure. The resulting mixture was quenched with deionised water and extracted with AcOEt $(3 \times 5 \mathrm{~mL})$. The organic phases were dried over $\mathrm{MgSO}_{4}$, followed by evaporation under reduced pressure to remove the solvent. The product was usually purified by column chromatography on silica gel (hexane/ethyl acetate) to give the corresponding products 3 .

2-Benzylbenzofuran (3a): ${ }^{[8]}$ Colorless oil; $t_{r}=14.2 ; R_{f}=0.6$ (hexane/ethyl acetate: 4/1); ${ }^{1} \mathrm{H}-\mathrm{NMR}\left(300 \mathrm{MHz}, \mathrm{CDCl}_{3}\right): \delta 7.40-7.45(\mathrm{~m}, 1 \mathrm{H}, \mathrm{ArH})$, 7.35-7.40 (m, 1H, ArH), 7.15-7.30 (m, 7H, ArH), $6.33(\mathrm{~s}, 1 \mathrm{H}, \mathrm{CH}), 4.06(\mathrm{~s}$, $\left.2 \mathrm{H}, \mathrm{CH}_{2}\right) ;{ }^{13} \mathrm{C}-\mathrm{NMR}\left(75 \mathrm{MHz} \mathrm{CDCl}_{3}\right): \delta 157.8,154.9,137.2,128.9(2 \mathrm{C})$, 128.8, 128.6 (2C), 126.7, 123.4, 122.5, 120.4, 110.9, 103.3, 35.0; IR (ATR): v 3029, 1454, 1252, $952 \mathrm{~cm}^{-1}$; MS (El) m/z (\%): $208\left(\mathrm{M}^{+}, 88\right), 207$ $\left(\mathrm{M}^{+}-1,100\right), 178(22), 131$ (36), 77 (8).

2-Benzyl-5,7-di-tert-butylbenzofuran (3b): Colorless oil; $t_{r}=17.0 ; R_{f}=$ 0.7 (hexane/ethyl acetate: 4/1); ${ }^{1} \mathrm{H}-\mathrm{NMR}\left(300 \mathrm{MHz}, \mathrm{CDCl}_{3}\right): \delta 7.31(\mathrm{~d}, \mathrm{~J}$ $=1.9 \mathrm{~Hz}, 1 \mathrm{H}, \mathrm{ArH}), 7.20-7.30(\mathrm{~m}, 5 \mathrm{H}, \mathrm{ArH}), 7.17(\mathrm{~d}, \mathrm{~J}=1.9 \mathrm{~Hz}, 1 \mathrm{H}, \mathrm{ArH})$, 
$6.31(\mathrm{~s}, 1 \mathrm{H}, \mathrm{CH}), 4.10\left(\mathrm{~s}, 2 \mathrm{H}, \mathrm{CH}_{2}\right), 1.47\left(\mathrm{~s}, 9 \mathrm{H}, \mathrm{CH}_{3} \times 3\right), 1.35(\mathrm{~s}, 9 \mathrm{H}$ $\left.\mathrm{CH}_{3} \times 3\right) ;{ }^{13} \mathrm{C}$-NMR $\left(75 \mathrm{MHz}, \mathrm{CDCl}_{3}\right): \delta 156.8,151.3,145.2,137.7$, $133.4,128.8$ (3C), 128.4 (2C), 126.5, 118.0, 114.5, 103.1, 35.1, 34.8, 34.4, 31.9 (3C), 29.9 (3C); IR (ATR): v 2955, 2905, 1603, 1479, 1242, $1030 \mathrm{~cm}^{-1}$.. MS (EI) m/z (\%): $321\left(\mathrm{M}^{+}+1,11\right), 320\left(\mathrm{M}^{+}, 49\right), 306(31), 305$ (100), 153 (9), 91 (17), 57 (12); HRMS calcd. (\%) for $\mathrm{C}_{23} \mathrm{H}_{28} \mathrm{O}$ : 320.21402; found: 320.2137 .

2-Benzyl-5-bromo-7-methoxybenzofuran (3c): Yellow solid; m.p. $=52-$ $54^{\circ} \mathrm{C}$ (Hexane); $\mathrm{r}_{\mathrm{r}}=17.6 ; \mathrm{R}_{\mathrm{f}}=0.7$ (hexane/ethyl acetate: $4 / 1$ ); ${ }^{1} \mathrm{H}-\mathrm{NMR}$ $\left(300 \mathrm{MHz}, \mathrm{CDCl}_{3}\right): \delta 7.20-7.35(\mathrm{~m}, 5 \mathrm{H}, \mathrm{ArH}), 7.18(\mathrm{~d}, \mathrm{~J}=1.7 \mathrm{~Hz}, 1 \mathrm{H}$, $\operatorname{ArH}), 6.83(\mathrm{~d}, \mathrm{~J}=1.7 \mathrm{~Hz}, 1 \mathrm{H}, \mathrm{ArH}), 6.24(\mathrm{t}, \mathrm{J}=0.94 \mathrm{~Hz}, 1 \mathrm{H}, \mathrm{CH}), 4.09(\mathrm{~s}$, $\left.2 \mathrm{H}, \mathrm{CH}_{2}\right), 3.95$ (s, 3H, OMe); ${ }^{13} \mathrm{C}-\mathrm{NMR}\left(75 \mathrm{MHz}, \mathrm{CDCl}_{3}\right)$ : $\delta 159.3,145.2$, 142.9, 136.7, 131.6, 128.9 (2C), 128.6 (2C), 126.8, 115.6 (2C), 109.3 , 103.2, 56.2, 34.8; IR (ATR): v 1597, 1472, $1207 \mathrm{~cm}^{-1}$; MS (EI) $\mathrm{m} / \mathrm{z}(\%)$ : $319\left(\mathrm{M}^{+}+3,17\right), 318\left(\mathrm{M}^{+}+2,96\right), 317\left(\mathrm{M}^{+}+1,57\right), 316\left(\mathrm{M}^{+}, 100\right), 315(21)$ 281 (19), 241 (10), 237 (19), 222 (16), 209 (11), 208 (19), 207 (41), 206 (91), 194 (19), 181 (37), 166 (19), 165 (64), 89 (14), 82 (12), 78 (44); HRMS calcd. (\%) for $\mathrm{C}_{16} \mathrm{H}_{13} \mathrm{BrO}_{2}$ : 316.0099; found: 316.0109 .

2-(2-Bromobenzyl)benzofuran (3d): Pale yellow oil; $t_{r}=16.0 ; R_{f}=0.6$ (hexane/ethyl acetate: $4 / 1) ;{ }^{1} \mathrm{H}-\mathrm{NMR}\left(300 \mathrm{MHz}, \mathrm{CDCl}_{3}\right): \delta 7.55-7.60(\mathrm{~m}$, $1 \mathrm{H}, \mathrm{ArH}), 7.45-7.50(\mathrm{~m}, 1 \mathrm{H}, \mathrm{ArH}), 7.40-7.45(\mathrm{~m}, 1 \mathrm{H}, \mathrm{ArH}), 7.10-7.30(\mathrm{~m}$, $5 \mathrm{H}, \mathrm{ArH}), 6.38(\mathrm{~s}, 1 \mathrm{H}, \mathrm{CH}), 4.24\left(\mathrm{~s}, 2 \mathrm{H}, \mathrm{CH}_{2}\right) ;{ }^{13} \mathrm{C}-\mathrm{NMR}(75 \mathrm{MHz}$ $\left.\mathrm{CDCl}_{3}\right): \delta 156.0,154.9,136.8,132.9,130.9,128.7,128.5,127.6,124.6$ 123.5, 122.6, 120.5, 110.9, 104.0, 35.2; IR (ATR): v 1601, 1585, 1453, 1251, $1025 \mathrm{~cm}^{-1}$; MS (EI) $\mathrm{m} / \mathrm{z}(\%): 289\left(\mathrm{M}^{+}+3,15\right), 288\left(\mathrm{M}^{+}+2,98\right), 287$ $\left(\mathrm{M}^{+}+1,54\right), 286\left(\mathrm{M}^{+}, 100\right), 285\left(\mathrm{M}^{+}-1,39\right), 208(11), 207(63), 206$ (17) 105 (41), 178 (73), 177 (16), 176 (24), 152 (16), 151 (12), 131 (82), 89 (33), 88 (11), 77 (14), 76 (26), 63 (12); HRMS calcd. (\%) for $\mathrm{C}_{15} \mathrm{H}_{11} \mathrm{BrO}$ 285.9993; found: 285.9985 .

2-(3-Chlorobenzyl)benzofuran (3e): $:^{[7]}$ Pale yellow oil; $t_{r}=15.5 ; R_{f}=0.6$ (hexane/ethyl acetate: $4 / 1) ;{ }^{1} \mathrm{H}-\mathrm{NMR}\left(300 \mathrm{MHz}, \mathrm{CDCl}_{3}\right)$ : $\delta$ 7.45-7.50 (m, $1 \mathrm{H}, \mathrm{ArH}), 7.35-7.40(\mathrm{~m}, 1 \mathrm{H}, \mathrm{ArH}), 7.29(\mathrm{br} \mathrm{s}, 1 \mathrm{H}, \mathrm{ArH}), 7.15-7.25(\mathrm{~m}, 5 \mathrm{H}$, $\mathrm{ArH}), 6.40(\mathrm{~s}, 1 \mathrm{H}, \mathrm{CH}), 4.06\left(\mathrm{~s}, 2 \mathrm{H}, \mathrm{CH}_{2}\right) ;{ }^{13} \mathrm{C}-\mathrm{NMR}\left(75 \mathrm{MHz}, \mathrm{CDCl}_{3}\right): \delta$ 156.7, 155.0, 139.2, 134.4, 129.8, 129.0, 128.6, 127.0 (2C), 123.6, 122.6 120.5, 110.9, 103.7, 34.6; IR (ATR): v 1596, 1585, 1574, 1453, 1252, $1008 \mathrm{~cm}^{-1} ; \mathrm{MS}(\mathrm{EI}) \mathrm{m} / \mathrm{z}(\%): 244\left(\mathrm{M}^{+}+2,34\right), 243\left(\mathrm{M}^{+}+1,37\right), 242\left(\mathrm{M}^{+}\right.$, 100), $241\left(\mathrm{M}^{+}-1,69\right), 208$ (10), 207 (77), 205 (13), 179 (22), 178 (44), 131 (67), 77 (13), 76 (14)

2-(4-Bromobenzyl)benzofuran (3f): $:^{[12]}$ White solid; m.p. $=47-49{ }^{\circ} \mathrm{C}$ (Hexane); $\mathrm{t}_{\mathrm{r}}=16.2 ; \mathrm{R}_{\mathrm{f}}=0.7$ (hexane/ethyl acetate: $4 / 1$ ); ${ }^{1} \mathrm{H}-\mathrm{NMR}(300$ $\mathrm{MHz}, \mathrm{CDCl}_{3}$ ): $\delta 7.40-7.50(\mathrm{~m}, 4 \mathrm{H}, \mathrm{ArH}), 7.10-7.20(\mathrm{~m}, 4 \mathrm{H}, \mathrm{ArH}), 6.36(\mathrm{dd}$ $\mathrm{J}=1.1,0.9 \mathrm{~Hz}, 1 \mathrm{H}, \mathrm{CH}), 4.03\left(\mathrm{~s}, 2 \mathrm{H}, \mathrm{CH}_{2}\right) ;{ }^{13} \mathrm{C}-\mathrm{NMR}\left(75 \mathrm{MHz}, \mathrm{CDCl}_{3}\right)$ : $\delta$ 156.9, 154.9, 136.2, 131.7 (2C), 130.6 (2C), 128.6, 123.6, 122.6, 120.7, $120.5,110.9,103.6,34.4$; IR (ATR): v 1599, 1584, 1488, 1452, 1250, $1010 \mathrm{~cm}^{-1}$; MS (EI) $\mathrm{m} / \mathrm{z}(\%): 289\left(\mathrm{M}^{+}+3,19\right), 288\left(\mathrm{M}^{+}+2,78\right), 287\left(\mathrm{M}^{+}+1\right.$ 69), $286\left(\mathrm{M}^{+}, 75\right), 285\left(\mathrm{M}^{+}-1,66\right), 208(19), 207$ (100), 205 (24), $179(25)$ 178 (56), 177 (16), 176 (14), 152 (14), 151 (11), 131 (52), 103 (12), 102 (13), 89 (25), 77 (17), 76 (32), 63 (12)

2-(4-Trifluoromethyl)benzyl)benzofuran (3g): ${ }^{[8]}$ Pale yellow oil; $t_{r}=$ 14.0; $\mathrm{R}_{\mathrm{f}}=0.6$ (hexane/ethyl acetate: $\left.4 / 1\right) ;{ }^{1} \mathrm{H}-\mathrm{NMR}\left(300 \mathrm{MHz}, \mathrm{CDCl}_{3}\right): \delta$ $7.58(\mathrm{~d}, J=7.8 \mathrm{~Hz}, 2 \mathrm{H}, \mathrm{ArH}), 7.45-7.50(\mathrm{~m}, 1 \mathrm{H}, \mathrm{ArH}), 7.40-7.45(\mathrm{~m}, 3 \mathrm{H}$, ArH), 7.15-7.25 (m, 2H, ArH), $6.41(\mathrm{~s}, 1 \mathrm{H}, \mathrm{CH}), 4.16\left(\mathrm{~s}, 2 \mathrm{H}, \mathrm{CH}_{2}\right) ;{ }^{13} \mathrm{C}-$ NMR $\left(75 \mathrm{MHz}, \mathrm{CDCl}_{3}\right): \delta 156.4,155.0,141.3,129.2(2 \mathrm{C}), 129.2$ (q, J $32.3 \mathrm{~Hz}), 128.1,126.0$ (q, $\mathrm{J}=272.4 \mathrm{~Hz}), 125.5(\mathrm{q}, \mathrm{J}=3.8 \mathrm{~Hz}, 2 \mathrm{C}), 123.7$ 122.7, 120.5, 110.9, 103.8, 34.8; IR (ATR): v 1454, 1322, $1252 \mathrm{~cm}^{-1}$; MS (EI) $\mathrm{m} / \mathrm{z}(\%): 277\left(\mathrm{M}^{+}+1,16\right), 276\left(\mathrm{M}^{+}, 100\right), 275\left(\mathrm{M}^{+}-1,84\right), 207(59)$, 179 (11), 178 (26), 131 (57)
2-(2-Methylbenzyl)benzofuran (3h): ${ }^{[7]}$ Pale yellow oil; $t_{r}=14.8 ; R_{f}=0.6$ (hexane/ethyl acetate: 4/1); ${ }^{1} \mathrm{H}$-NMR $\left(300 \mathrm{MHz}, \mathrm{CDCl}_{3}\right.$ ): $\delta 7.42$ (ddd, J = 8.2, 1.8, 0.7 Hz, 2H, ArH), 7.10-7.25 (m, $6 \mathrm{H}, \mathrm{ArH}), 6.23(\mathrm{~d}, \mathrm{~J}=0.9 \mathrm{~Hz}, 1 \mathrm{H}$, $\mathrm{CH}), 4.09\left(\mathrm{~s}, 2 \mathrm{H}, \mathrm{CH}_{2}\right), 2.34\left(\mathrm{~s}, 3 \mathrm{H}, \mathrm{CH}_{3}\right) ;{ }^{13} \mathrm{C}-\mathrm{NMR}\left(75 \mathrm{MHz}, \mathrm{CDCl}_{3}\right): \delta$ $157.5,154.9,136.6,135.4,130.4,129.8,128.8,127.1,126.2,123.3$ 122.5, 120.3, 110.9, 103.2, 32.7, 19.4; IR (ATR): v 1599, 1585, 1454 1253, $1008 \mathrm{~cm}^{-1}$; MS (EI) $\mathrm{m} / \mathrm{z}(\%): 223\left(\mathrm{M}^{+}+1,18\right), 222\left(\mathrm{M}^{+}, 100\right), 221$ (M+1, 31), 207 (37), 178 (23), 131 (27), 116 (11), 115 (10), 110 (10), 107 (48), 104 (22), 77 (11).

2-(3-Methylbenzyl)benzofuran (3i) ${ }^{[8]}$ Colorless oil; $t_{r}=14.7 ; R_{f}=0.7$ (hexane/ethyl acetate: $4 / 1) ;{ }^{1} \mathrm{H}-\mathrm{NMR}\left(300 \mathrm{MHz}, \mathrm{CDCl}_{3}\right)$ : $\delta$ 7.45-7.50 (m, $1 \mathrm{H}, \mathrm{ArH}), 7.40-7.45(\mathrm{~m}, 1 \mathrm{H}, \mathrm{ArH}), 7.15-7.25(\mathrm{~m}, 3 \mathrm{H}, \mathrm{ArH}), 7.05-7.10(\mathrm{~m}$ $3 \mathrm{H}, \mathrm{ArH}), 6.37(\mathrm{~s}, 1 \mathrm{H}, \mathrm{CH}), 4.06\left(\mathrm{~s}, 2 \mathrm{H}, \mathrm{CH}_{2}\right), 2.33\left(\mathrm{~s}, 3 \mathrm{H}, \mathrm{CH}_{3}\right) ;{ }^{13} \mathrm{C}-$ $\mathrm{NMR}\left(75 \mathrm{MHz}, \mathrm{CDCl}_{3}\right): \delta 157.9,154.9,138.2,137.1,129.6,128.8,128.5$, $127.5,125.9,123.3,122.5,120.4,110.9,103.3,34.9,21.4$; IR (ATR): v 3065, 3026, 1601, 1586, 1454, 1251, $954 \mathrm{~cm}^{-1}$; MS (EI) $\mathrm{m} / \mathrm{z}(\%): 222\left(\mathrm{M}^{+}\right.$, 100), 221 ( $\left.M^{+}-1,81\right), 207$ (54), 179 (11), 178 (25), 131 (34)

2-(4-Methoxybenzyl)benzofuran (3j): ${ }^{[8]}$ Colorless oil; $t_{r}=15.9 ; R_{f}=0.5$ (hexane/ethyl acetate: $4 / 1) ;{ }^{1} \mathrm{H}-\mathrm{NMR}\left(300 \mathrm{MHz}, \mathrm{CDCl}_{3}\right)$ : $\delta$ 7.45-7.50 (m, $1 \mathrm{H}, \mathrm{ArH}), 7.35-7.40(\mathrm{~m}, 1 \mathrm{H}, \mathrm{ArH}), 7.15-7.25(\mathrm{~m}, 4 \mathrm{H}, \mathrm{ArH}), 6.85-6.90(\mathrm{~m}$ $2 \mathrm{H}, \mathrm{ArH}), 6.34(\mathrm{dd}, \mathrm{J}=1.9,0.9 \mathrm{~Hz}, 1 \mathrm{H}, \mathrm{CH}), 4.04\left(\mathrm{~s}, 2 \mathrm{H}, \mathrm{CH}_{2}\right), 3.79(\mathrm{~s}$, $\left.3 \mathrm{H}, \mathrm{OCH}_{3}\right) ;{ }^{13} \mathrm{C}-\mathrm{NMR}\left(75 \mathrm{MHz}, \mathrm{CDCl}_{3}\right): \delta 158.5,158.3,154.9,129.9$ (2C), 129.3, 128.8, 123.3, 122.5, 120.4, 114.0 (2C), 110.9, 103.1, 55.3, 34.1; IR (ATR): v 1612, 1584, 1509, $1245 \mathrm{~cm}^{-1}$; MS (EI) $\mathrm{m} / \mathrm{z}(\%): 238\left(\mathrm{M}^{+}\right.$, 59), $237\left(\mathrm{M}^{+}-1,56\right), 207$ (100), 131 (13).

2-Hexylbenzofuran (3k): ${ }^{[6 c]}$ Colorless oil; $t_{r}=12.6 ; R_{f}=0.9$ (hexane/ethyl acetate: 4/1); ${ }^{1} \mathrm{H}-\mathrm{NMR}\left(300 \mathrm{MHz}, \mathrm{CDCl}_{3}\right)$ : $\delta$ 7.35-7.40, 7.45-7.50 (2m, $1 \mathrm{H}$ and $1 \mathrm{H}$ respectively, $\mathrm{CH} \times 2), 7.10-7.20(\mathrm{~m}, 2 \mathrm{H}, \mathrm{CH} \times 2), 6.34(\mathrm{~s}, 1 \mathrm{H}$, $\mathrm{OCCH}$ ), 2.74 (t, $J=7.5 \mathrm{~Hz}, 2 \mathrm{H}, \mathrm{CH}_{2}$ ), 1.73 (quin-, $\mathrm{J}=7.5 \mathrm{~Hz}, 2 \mathrm{H}, \mathrm{CH}_{2}$ ), 1.25-1.40 (m, 6H, CH $\times 3), 0.89\left(\mathrm{t}, \mathrm{J}=7.14 \mathrm{~Hz}, 3 \mathrm{H}, \mathrm{CH}_{3}\right) ;{ }^{13} \mathrm{C}-\mathrm{NMR}(75$ $\left.\mathrm{MHz}, \mathrm{CDCl}_{3}\right): \delta 159.8,154.7,129.1,123.0,122.4,120.2,110.7,101.8$, 31.6, 28.9, 28.5, 27.7, 22.6, 14.1; IR (ATR): v 1600, 1587, 1252, 1009 $738 \mathrm{~cm}^{-1}$; MS (EI) m/z (\%): $202\left(\mathrm{M}^{+}, 24\right), 132$ (29), 131 (100), 95 (13), 77 (13).

2-(Cyclohexylmethyl)benzofuran (3I): Colorless oil; $\mathrm{t}_{\mathrm{r}}=13.9 ; \mathrm{R}_{\mathrm{f}}=0.7$ (hexane/ethyl acetate: $4 / 1)$; ${ }^{1} \mathrm{H}-\mathrm{NMR}\left(300 \mathrm{MHz}, \mathrm{CDCl}_{3}\right): \delta 7.35-7.50(\mathrm{~m}$, $2 \mathrm{H}, \mathrm{ArH}), 7.10-7.20(\mathrm{~m}, 2 \mathrm{H}, \mathrm{ArH}), 6.35(\mathrm{~s}, 1 \mathrm{H}, \mathrm{CH}), 2.63(\mathrm{~d}, \mathrm{~J}=6.8 \mathrm{~Hz}$ $\left.2 \mathrm{H}, \mathrm{CH}_{2}\right), 1.60-1.80\left(\mathrm{~m}, 6 \mathrm{H}, \mathrm{CH}_{2} \times 3\right), 1.10-1.30\left(\mathrm{~m}, 3 \mathrm{H}, \mathrm{CH}_{2}, \mathrm{CH}\right), 0.90-$ $1.05\left(\mathrm{~m}, 2 \mathrm{H}, \mathrm{CH}_{2}\right) ;{ }^{13} \mathrm{C}-\mathrm{NMR}\left(75 \mathrm{MHz}, \mathrm{CDCl}_{3}\right)$ : $\delta$ 158.5, 154.7, 129.0, $122.9,122.3,120.1,110.7,102.8,37.0,36.3,33.2(2 \mathrm{C}), 26.4,26.2(2 \mathrm{C})$ IR (ATR): v 2921, 2850, 1601, 1586, 1453, 1253, $1008 \mathrm{~cm}^{-1}$; MS (EI) $\mathrm{m} / \mathrm{z}$ (\%): $214\left(\mathrm{M}^{+}, 50\right), 133$ (12), 132 (90), 131 (100), 83 (10), 77 (10); HRMS calcd. (\%) for $\mathrm{C}_{15} \mathrm{H}_{18} \mathrm{O}$ : 214.1357; found: 214.1351 .

2-(4-Chlorobutyl)benzofuran (3m) ${ }^{[13]}$ Colorless oil; $\mathrm{t}_{\mathrm{r}}=13.2 ; \mathrm{R}_{\mathrm{f}}=0.7$ (hexane/ethyl acetate: $4 / 1) ;{ }^{1} \mathrm{H}-\mathrm{NMR}\left(300 \mathrm{MHz}, \mathrm{CDCl}_{3}\right)$ : $\delta$ 7.45-7.50 (m, $1 \mathrm{H}, \mathrm{ArH}), 7.40-7.45(\mathrm{~m}, 1 \mathrm{H}, \mathrm{ArH}), 7.15-7.25(\mathrm{~m}, 2 \mathrm{H}, \mathrm{ArH}), 6.40(\mathrm{~s}, 1 \mathrm{H}$ $\mathrm{CH}$ ), $3.58\left(\mathrm{t}, \mathrm{J}=6.2 \mathrm{~Hz}, 2 \mathrm{H}, \mathrm{CH}_{2}\right), 2.81\left(\mathrm{t}, \mathrm{J}=7.1 \mathrm{~Hz}, 2 \mathrm{H}, \mathrm{CH}_{2}\right)$, $1.85-$ $1.95\left(\mathrm{~m}, 4 \mathrm{H}, \mathrm{CH}_{2} \times 2\right) ;{ }^{13} \mathrm{C}-\mathrm{NMR}\left(75 \mathrm{MHz}, \mathrm{CDCl}_{3}\right): \delta 158.6,154.6,128.8$, 123.2, 122.5, 120.2, 110.7, 102.2, 44.6, 31.9, 27.7, 25.0; IR (ATR): v 2922, 2853, 1603, 1584, 1455, 1252, $948 \mathrm{~cm}^{-1}$; MS (EI) $\mathrm{m} / \mathrm{z}(\%): 208\left(\mathrm{M}^{+}\right.$, 18), 132 (15), 131 (100), 77 (10).

2-(2-(Tetrahydro-2H-pyran-2-yl)oxy)ethyl)benzofuran (3n): Colorless oil; $\mathrm{t}_{\mathrm{r}}=15.0 ; \mathrm{R}_{\mathrm{f}}=0.5$ (hexane/ethyl acetate: $\left.4 / 1\right) ;{ }^{1} \mathrm{H}-\mathrm{NMR}(300 \mathrm{MHz}$ $\left.\mathrm{CDCl}_{3}\right): \delta$ 7.45-7.50 (m, $\left.1 \mathrm{H}, \mathrm{ArH}\right), 7.35-7.40(\mathrm{~m}, 1 \mathrm{H}, \mathrm{ArH}), 7.15-7.25(\mathrm{~m}$, $2 \mathrm{H}, \mathrm{ArH}), 6.47(\mathrm{~d}, \mathrm{~J}=0.9 \mathrm{~Hz}, 1 \mathrm{H}, \mathrm{CH}), 4.60-4.65(\mathrm{~m}, 1 \mathrm{H}, \mathrm{CH}), 4.09(\mathrm{dt}, \mathrm{J}$ $=9.8,6.8 \mathrm{~Hz}, 1 \mathrm{H}, \mathrm{CH}), 3.75-3.85\left(\mathrm{~m}, 2 \mathrm{H}, \mathrm{CH}_{2}\right), 3.45-3.55(\mathrm{~m}, 1 \mathrm{H}, \mathrm{CH})$, $3.08\left(\mathrm{td}, \mathrm{J}=6.8,0.8 \mathrm{~Hz}, 2 \mathrm{H}, \mathrm{CH}_{2} \mathrm{CH}_{2} \mathrm{O}\right), 1.45-1.85\left(\mathrm{~m}, 6 \mathrm{H}, \mathrm{CH}_{2} \mathrm{x}\right.$ 
3): ${ }^{13} \mathrm{C}-\mathrm{NMR}\left(75 \mathrm{MHz}, \mathrm{CDCl}_{3}\right): \delta 156.4,154.6,128.9,123.2,122.4$, 120.3, 110.7, 103.0, 98.8, 65.0, 62.2, 30.6, 29.3, 25.4, 19.4; IR (ATR): v 2941, 2871, 1602, 1587, 1455, 1252, $1030 \mathrm{~cm}^{-1}$; MS (EI) $\mathrm{m} / \mathrm{z}$ (\%): 246 $\left.\left(\mathrm{M}^{+}, 1\right), 162(28), 145813\right), 144(50), 132(16), 131$ (100), $85(26), 77$ (14), 55 (12); HRMS calcd. (\%) for $\mathrm{C}_{15} \mathrm{H}_{18} \mathrm{O}_{3}$ : 246.1256; found: 246.1264

2-(3-Ethylbenzyl)benzofuran (3o): Pale yellow oil; $t_{r}=15.4 ; R_{f}=0.7$ (hexane/ethyl acetate: $4 / 1) ;{ }^{1} \mathrm{H}-\mathrm{NMR}\left(300 \mathrm{MHz}, \mathrm{CDCl}_{3}\right): \delta$ 7.35-7.50 (m, $4 \mathrm{H}, \mathrm{ArH}), 7.25-7.30(\mathrm{~m}, 2 \mathrm{H}, \mathrm{ArH}), 7.15-7.20(\mathrm{~m}, 2 \mathrm{H}, \mathrm{ArH}), 6.39(\mathrm{~d}, \mathrm{~J}=0.9$ $\mathrm{Hz}, 1 \mathrm{H}, \mathrm{CH}), 4.08\left(\mathrm{~s}, 2 \mathrm{H}, \mathrm{CH}_{2}\right), 3.06(\mathrm{~s}, 1 \mathrm{H}, \mathrm{CH}) ;{ }^{13} \mathrm{C}-\mathrm{NMR}(75 \mathrm{MHz}$, $\left.\mathrm{CDCl}_{3}\right): \delta 157.0,155.0,144.9,137.5,132.5,130.6,129.5,128.6,123.6$, 122.6, 120.5, 110.9, 103.6, 83.5, 77.3, 34.7; IR (ATR): v 3291, 1599, 1584, 1454, 1251, $1008 \mathrm{~cm}^{-1}$; MS (EI) $\mathrm{m} / \mathrm{z}(\%): 233\left(\mathrm{M}^{+}+1,18\right), 232\left(\mathrm{M}^{+}\right.$, 100), 231 ( $\left.\mathrm{M}^{+}-1,87\right), 203$ (14), 202 (31), 131 (36), 101 (11); HRMS calcd. (\%) for $\mathrm{C}_{17} \mathrm{H}_{12} \mathrm{O}$ : 232.0888; found: 232.0886 .

2-(4-Bromobenzyl)-5,7-di-tert-butylbenzofuran (3p): White solid; m.p. $=104-106^{\circ} \mathrm{C}\left(\right.$ Hexane); $\mathrm{t}_{\mathrm{r}}=15.0 ; \mathrm{R}_{\mathrm{f}}=0.8$ (hexane/ethyl acetate: $\left.4 / 1\right) ;{ }^{1} \mathrm{H}-$ NMR (300 MHz, $\left.\mathrm{CDCl}_{3}\right): \delta 7.40-7.45(\mathrm{~m}, 2 \mathrm{H}, \mathrm{ArH}), 7.32(\mathrm{~d}, \mathrm{~J}=1.9 \mathrm{~Hz}$ $1 \mathrm{H}, \mathrm{ArH}), 7.15-7.20(\mathrm{~m}, 3 \mathrm{H}, \mathrm{ArH}), 6.32(\mathrm{~s}, 1 \mathrm{H}, \mathrm{CH}), 4.05\left(\mathrm{~s}, 2 \mathrm{H}, \mathrm{CH}_{2}\right)$ $1.46\left(\mathrm{~s}, 9 \mathrm{H}, \mathrm{CH}_{3} \times 3\right), 1.35\left(\mathrm{~s}, 9 \mathrm{H}, \mathrm{CH}_{3} \times 3\right) ;{ }^{13} \mathrm{C}-\mathrm{NMR}\left(75 \mathrm{MHz}, \mathrm{CDCl}_{3}\right)$ : ס155.9, 151.4, 145.4, 136.7, 133.5, 131.5 (2C), 130.5 (2C), 126.7, 120.4, 118.2, 114.6, 103.4, 34.8, 34.5, 34.4, 31.9 (3C), 29.9 (3C); IR (ATR): v 2959, 2950, 1607, $1478 \mathrm{~cm}^{-1}$; MS (EI) $\mathrm{m} / \mathrm{z}(\%): 401\left(\mathrm{M}^{+}+2,39\right), 400$ $\left(\mathrm{M}^{+}+1,10\right), 399\left(\mathrm{M}^{+}, 39\right), 386(22), 385(99), 384(24), 383(100), 281$ (10), 227 (12), 207 (28), 169 (16), 152 (10), 138 (56), 57 (46); HRMS calcd. (\%) for $\mathrm{C}_{23} \mathrm{H}_{27} \mathrm{BrO}$ : 398.1245; found: 398.1251

\section{General procedure for the preparation of the products 5 .}

To a stirred solution of the corresponding acetophenone $(4,0.4 \mathrm{mmol})$ in $\mathrm{EtOH}(2 \mathrm{~mL})$ was added 4-methylbenzenesulfonohydrazide $(74 \mathrm{mg}, 0.4$ $\mathrm{mmol}$ ) and the reaction was stirred at $100{ }^{\circ} \mathrm{C}$ during $1 \mathrm{~h}$. After that time, $\mathrm{Cs}_{2} \mathrm{CO}_{3}\left(390 \mathrm{mg}, 1.2 \mathrm{mmol}\right.$ ), $\mathrm{CuO}-\mathrm{Fe}_{3} \mathrm{O}_{4}(50 \mathrm{mg}, 2.4 \mathrm{~mol} \%$ ) were added to the reaction solution followed by the corresponding terminal alkyne $(2,0.5 \mathrm{mmol})$. The resulting mixture was stirred at $100^{\circ} \mathrm{C}$ during 5 hours. The catalyst was removed by a magnetic decantation and the solvent was removed under reduce pressure. The resulting mixture was quenched with deionised water and extracted with AcOEt $(3 \times 5 \mathrm{~mL})$. The organic phases were dried over $\mathrm{MgSO}_{4}$, followed by evaporation under reduced pressure to remove the solvent. The product was usually purified by chromatography on silica gel (hexane/ethyl acetate) to give the corresponding products 5

2-Benzyl-3-methylbenzofuran (5a) ${ }^{[14]}$ Colorless oil; $t_{r}=14.4 ; R_{f}=0.7$ (hexane/ethyl acetate: $4 / 1) ;{ }^{1} \mathrm{H}-\mathrm{NMR}\left(300 \mathrm{MHz}, \mathrm{CDCl}_{3}\right): \delta 7.45-7.50(\mathrm{~m}$, $1 \mathrm{H}, \mathrm{ArH}), 7.20-7.40(\mathrm{~m}, 8 \mathrm{H}, \mathrm{ArH}), 4.09\left(\mathrm{~s}, 2 \mathrm{H}, \mathrm{CH}_{2}\right), 2.23(\mathrm{~s}, 3 \mathrm{H}$, $\left.\mathrm{CH}_{3}\right) ;{ }^{13} \mathrm{C}$-NMR $\left(75 \mathrm{MHz}, \mathrm{CDCl}_{3}\right): \delta 154.0,152.1,138.0,130.2,128.6$ (2C), 128.5 (2C), 126.5, 123.4, 122.0, 118.9, 110.8, 32.6, 8.0; IR (ATR): v 1494, 1454, 1088, $744 \mathrm{~cm}^{-1}$; MS (EI) $\mathrm{m} / \mathrm{z}(\%): 223\left(\mathrm{M}^{+}+1,19\right), 222\left(\mathrm{M}^{+}\right.$, 94), 221 (M+1, 49), 208 (23), 207 (100), 178 (20), 145 (48), 131 (10), 115 (19).

2-Benzyl-3-methylbenzofuran (5b): Colorless oil; $\mathrm{t}_{\mathrm{r}}=16.5 ; \mathrm{R}_{\mathrm{f}}=0.7$ (hexane/ethyl acetate: $4 / 1) ;{ }^{1} \mathrm{H}-\mathrm{NMR}\left(300 \mathrm{MHz}, \mathrm{CDCl}_{3}\right): \delta 7.56$ (d, $\mathrm{J}=$ $1.9 \mathrm{~Hz}, 1 \mathrm{H}, \mathrm{ArH}), 7.25-7.30(\mathrm{~m}, 3 \mathrm{H}, \mathrm{ArH}), 7.20-7.25(\mathrm{~m}, 4 \mathrm{H}, \mathrm{ArH}), 4.07(\mathrm{~s}$ $\left.2 \mathrm{H}, \mathrm{CH}_{2}\right), 2.18\left(\mathrm{~s}, 3 \mathrm{H}, \mathrm{CH}_{3}\right) ;{ }^{13} \mathrm{C}-\mathrm{NMR}\left(75 \mathrm{MHz}, \mathrm{CDCl}_{3}\right): \delta 153.6,152.8$, $137.5,132.3,128.6(2 \mathrm{C}), 128.4(2 \mathrm{C}), 126.6,126.2,121.7,115.2,112.2$ 110.5, 32.6, 7.9; IR (ATR): v 1602, 1494, 1445, 1262, 1089, $799 \mathrm{~cm}^{-1}$ MS (EI) $\mathrm{m} / \mathrm{z}(\%): 302\left(\mathrm{M}^{+}+2,72\right), 301\left(\mathrm{M}^{+}+1,38\right), 300\left(\mathrm{M}^{+}, 80\right), 299\left(\mathrm{M}^{+}\right.$ 1, 28), 287 (85), 285 (100), 281 (33), 225 (37), 223 (27), 207 (99), 205 (27), 193 (29), 191 (18), 115 (28), 110 (18), 103 (27), 89 (21), 88 (16), 78
(17), 74 (17), 73 (16), 63 (19), 61 (23). HRMS calcd. (\%) for $\mathrm{C}_{16} \mathrm{H}_{13} \mathrm{BrO}$ : 300.0150; found: 300.0143

\section{Acknowledgements}

This work was supported by the Spanish Ministerio de Economía y Competitividad (MICINN; CTQ2011-24151 and CTQ2015-66624-P) and University of Alicante. J.M.P. thanks the MICINN (FPI program) for her fellowship.

Keywords: Nanoparticles • Heterogeneous catalysis • Alkynes • Copper • Fused-ring systems

[1] R. Benassi, in Comprehensive Heterocyclic Chemistry II, Vol. 2, ch 2.05, (Eds: A. R. Katritzky, C. W. Rees, E. F. V. Scriven), Pergamon Press, Oxford, 1996, 259-295.

[2] a) B. A. Keay, in Comprehensive Heterocyclic Chemistry II, Vol. 2, ch 2.08, (Eds: A. R. Katritzky, C. W. Rees, E. F. V. Scriven), Pergamon Press, Oxford, 1996, pp. 395-435; b) A. Radadiya, A. Shah, Eur. J. Med. Chem. 2015, 97, 356-376; c) H. Khanam, Shamsuzzaman, Eur. J. Med. Chem. 2015, 97, 483-504.

[3] a) H. Heaney, J. S. Ahn, in Comprehensive Heterocyclic Chemistry II, Vol. 2, ch. 2.06, (Eds: A. R. Katritzky, C. W. Rees, E. F. V. Scriven) Pergamon Press, Oxford, 1996, pp. 297-348; b) A. A. Abu-Hashem, H. A. R. Hussein, A. S. Aly, M. A. Gouda, Synth. Commun. 2014, 44 2899-2920.

[4] a) W. Friedrichsen, in Comprehensive Heterocyclic Chemistry II, Vol. 2, ch. 2.07, (Eds.: A. R. Katritzky, C. W. Rees, E. F. V. Scriven), Pergamon Press, Oxford, 1996, pp. 352-392; b) C. P. Dell, in Science of Synthesis, Vol. 10, ch. 1, (Eds: E. J. Thomas), Georg Thieme Verlag, Stuttgart, 2002, 1, pp. 11-86; c) A. A. Abu-Hashem, H. A. R. Hussein, A S. Aly, M. A. Gouda, Synth. Commun. 2014, 44, 2285-2312; d) S Cacchi, G. Fabrizi, A. Goggiamani, Org. Biomol. Chem. 2011, 9, 641 652.

[5] a) E. J. Guthrie, J. Macritchie, R. C. Hartley, Tetrahedron Lett. 2000, 41, 4987-4990; b) T. Pei, C.-Y. Chen, L. CiMichele, I. W. Davies, Org. Lett 2010, 12, 4972-4975; c) S. Ghosh, J. Das, Tetrahedron Lett. 2011, 52 1112-1116; d) J. Liu, Z. Liu, P. Liao, X. Bi, Org. Lett. 2014, 16, 62046207.

[6] a) C. G. Bates, P. Saejueng, J. M. Murphy, D. Venkataraman, Org Chem. 2002, 4, 4727-4729; b) M. Nagamochi, Y.-Q. Fang, M. Lautens Org. Lett. 2007, 9, 2955-2958; c) R. Cano, M. Yus, D. J. Ramón Tetrahedron 2012, 68, 1393-1400; d) C. Rossy, E. Fouquet, F.-X. Felpin, Beilstein J. Org. Chem. 2013, 9, 1426-1431.

[7] T. Xiao, X. Dong, L. Zhou, Org. Biomol. Chem. 2013, 11, 1490-1497.

[8] L. Zhou, Y. Shi, Q. Xiao, Y. Liu, F. Ye, Y. Zhang, J. Wang, Org. Lett. 2011, 13, 968-971.

[9] a) S. Hyun, T. Ko, K. Han, J. H. Oh, Phys. Stat. Sol. 2004, 1, 3468 3471; b) M. J. Aliaga, D. J. Ramón, M. Yus, Org. Biomol. Chem. 2010 8, 43-46; c) R. Cano, D. J. Ramón, M. Yus, J. Org. Chem. 2010, 75 3458-3460; d) R. Cano, M. Yus, D. J. Ramón, Tetrahedron 2012, 68 1393-1400; e) J. M. Pérez, R. Cano, M. Yus, D. J. Ramón, Synthesis 2013, 45, 1373-1379; Erratum: J. M. Pérez, R. Cano, M. Yus, D. J. Ramón, Synthesis 2013, 45, 2768; f) J. M. Pérez, R. Cano, M. Yus, D. J. Ramón, Eur. J. Org. Chem. 2012, 4548-4554; g) J. M. Pérez, R Cano, D. J. Ramón, RSC Adv. 2014, 4, 23943-23951; h) S. N Shelke, S. R. Bankar, G. R. Mhaske, S. S. Kadam, D. K. Murade, S. B. Bhorkade, A. K. Rathi, N. Bundaleski, O. M. N. D. Teodoro, R. Z. Zboril, R. S. Varma, M. B. Gawande, ACS Sustainable Chem. Eng. 2014, 2 , 1699-1706; i) R. K. Sharma, R. Gaur, M. Yadav, A. K. Rathi, J. Pechousek, M. Petr, R. Zboril, M. G. Gawande, ChemCatChem, 2015 
7, 3495-3502. j) X. Marset, J. M. Pérez, D. J. Ramón, Green Chem 2016, 18, 826-833

[10] a) M. B. Gawande, P. S. Branco, R. S. Varma, Chem. Soc. Rev. 2013 42, 3371-3393; b) D. Wang, D. Astruc, Chem. Rev. 2014, 114, 69496985; c) M. B. Gawande, R. Luque, R. Zboril, ChemCatChem 2014, 6 3312-3313; d) D. J. Ramón, Johnson Matthey Technol. Rev. 2015, 59, 120-122; e) A. Baeza, G. Guillena, D. J. Ramón, ChemCatChem 2016, 8, 49-67; f) R. K. Sharma, S. Dutta, S. Sharma, R. Zboril, R. S. Varma M. B. Gawande, Green Chem. 2016, 18, 3184-3209; g) M. B. Gawande A. Goswami, F.-X. Felpin, T. Asefa, X. Huang, R. Silva, X. Zou, R. Zboril, R. S. Varma, Chem. Rev. 2016, 116, 3722-3811.
[11] For an example were recyclable impregnated cobalt oxide on magnetite acts as heterogeneous reservoir for the homogeneous reaction, see: $\mathrm{J}$. M. Pérez, D. J. Ramón, Adv. Synth. Catal. 2014, 356, 3039-3047.

[12] J. Barluenga, M. Tomás-Gamasa, F. Aznar, C. Valdés, Nat. Chem 2009, 1, 494-499.

[13] M. Yamaguchi, H. Katsumata, K. Manabe, J. Org. Chem. 2013, 78 9270-9281.

[14] R. Stoermer, R. Wehln, Ber. Dtsch. Chem. Ges. 1902, 35, 3549-3560 


\section{Entry for the Table of Contents}

\section{FULL PAPER}

An efficient direct transformation of $o$ hydroxybenzaldehydes into the corresponding benzo[ $b]$ furans is introduced using alkynes and catalyzed by a heterogeneous copper oxide impregnated on magnetite.

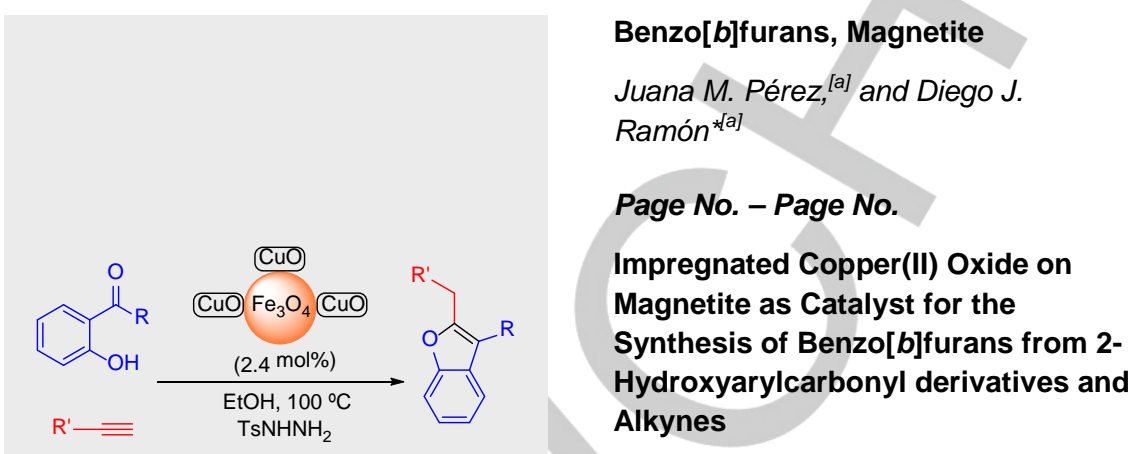

